\title{
Esquemas de gênero e perfil idiocêntrico e alocêntrico dos lutadores de jiu-jitsu de Chapecó-SC
}

\author{
Gender and idiocentricand alocentric schemes profile \\ of the ilu-itsu fighters from Chapeco-5C
}

Mariluce Poerschke Vieira, ${ }^{1}$ Kelson Mathiola Backes, ${ }^{1}$ Walan Robert da Silva, ${ }^{2}$ Gislane Ferreira de Melo, ${ }^{3}$ Fernando Luiz Cardoso 2

'Universidade do Oeste de Santa Catarina (UNOESC), Chapecó, SC, Brasil.

¿Universidade do Estado de Santa Catarina (UDESC), Florianópolis, SC, Brasil.

3Universidade Católica de Brasília (UCB), Brasília, DF, Brasil.

Recebido em: 12/07/2016 / Aceito em: 22/08/2016 / Publicado em: 30/09/2016

marilucekuty@hotmail.com

\section{RESUMO}

Objetivo: investigar qual o perfil psicológico dos atletas de jiu-jitsu de Chapecó-SC, em relação aos esquemas de gênero (EG) e o perfil idiocêntrico e alocêntrico (PI-A). Método: participaram da pesquisa 32 praticantes de jiu-jitsu, do sexo masculino, jovens e adultos com média de idade igual a 27,5 anos (DP= $6,10)$. Foi aplicado um questionário para verificar as características sociodemográficas, econômicas e esportivas dos atletas, juntamente com o Inventário Masculino dos Esquemas de Gênero do Autoconceito (IMEGA) e o Inventário de Perfil Idiocêntrico-Alocêntrico (PI-A). Resultados: pode-se observar que a maioria dos atletas vive com uma companheira, se declararam de cor branca e pertencentes à classe média. Em relação aos esquemas de gênero, 62,5\% dos atletas classificaram-se em um perfil isoesquemático, apresentando assim, uma simetria entre os esquemas masculino e feminino. Referindo-se ao perfil idiocêntrico-alocêntrico, $65.6 \%$ classificaram-se como alocêntricos, o que demonstra que os atletas são mais coletivistas do que individualistas. Considerações finais: os atletas de jiu-jitsu possuem um perfil de gênero adequado à prática desportiva e que apesar de pertencerem a uma modalidade individual, apresentam uma predominância alocêntrica em relação ao seu comportamento. Sugere-se que este perfil mais coletivista se deva a estrutura organizacional da competição que valoriza uma articulação entre a pontuação do individuo e do grupo.

Palavras-chave: Perfil Psicológico; Gênero; Tracos; Personalidade.

\section{ABSTRACT}

Objective: investigated the profile of Jiu-Jitsu athletes from Chapecó-SC in relation to gender schemas (GS) and idiocêntrico and allocentric profile (I-AP). Method: the study sample consisted of 32 youth adults males 'practitioners of jiu-jitsu with mean age of 27.5 years $(S D=6.10)$. I $t$ was applied a questionnaire to measure the athletes' demographic, economic and sporting characteristics, simultaneously with the Self Inventory of Male Gender Schemas (IMEGA) and the Idiocêntrico-allocentric profile (I-AP). Results: it can be seen that the majority of athletes live as a couple and self-declared as white and belong to the middle class. Regarding gender schemes, $62.5 \%$ of the athletes were classified as isoesquemático profile, thus presenting a symmetry between masculine and feminine schemes. Referring to idiocentric and allocentric-profile, 65.6\% were classified as alocentrics, which shows that athletes are more collectivist than individualist. Closing remarks: that the jiu-jitsu athletes have an adequate gender profile sports practice and despite belonging to an individual sport, present a predominance alocentric in relation behavior. It is suggested that this more collectivist profile is due to the organizational structure of the competition that values a link between the scores of the individual and the group.

Keywords: Profile Psychology; Gender; Tracers; Personality. 


\section{INTRODUÇÃO}

A psicologia do esporte é vista como uma ciência que estuda as condutas e os processos psíquicos de atletas, acelerando os processos naturais de desenvolvimento das qualidades psíquicas e propriedades da personalidade mais relevantes aos esportistas. Os estudos nesta área acontecem por meio do conhecimento das condições internas dos atletas e tem como finalidade otimizar o potencial físico, técnico e tático dos mesmos, adquiridos durante o processo de preparação psicológica. ${ }^{1}$

Dentro desta ótica, o atleta de alto rendimento é moldado de acordo com as variáveis psicológicas e sociais, formando uma personalidade dissociada da definição de gênero concebido histórico-filosoficamente. ${ }^{2}$ Diante disso, Bara Filho, Ribeiro ${ }^{3}$ destacam que as características psicológicas dos atletas de alto rendimento são especiais e diferem entre as modalidades esportivas, o que se deve às situações específicas geradas, durante os treinos e competições.

Tendo em vista as especificidades encontradas nas modalidades individuais, este estudo buscou o jiu-jítsu por ser uma luta praticada há pelo menos dois mil anos e considerada um dos mais antigos exercícios físicos do mundo, se mantendo até os dias de hoje. Esta modalidade de arte marcial iniciou-se com o objetivo de defesa pessoal e hoje é considerada como um esporte que consiste em basicamente de lutas com quedas, imobilizações e golpes ${ }^{4}$ na qual o praticante deve manter um controle físico direto sobre o adversário e dominá-lo com sua própria força.

Além dos benefícios que a prática regular das artes marciais transmitem para o ser humano, tanto na infância como na fase adulta, o jiu-jitsu procura resgatar o conceito de disciplina e respeito de um indivíduo para outro exclusivamente pelo meio em que vive, contribuindo para formação de caráter e promoção a saúde, ${ }^{5}$ fatores de extrema importância no desempenho do atleta. ${ }^{6}$

No entanto, quando analisado os aspectos psicológicos dos lutadores, as situações geradas durante os treinamentos e competições podem estar vinculadas aos esquemas de gênero, os quais são partes constitutivas do autoconceito, estando relacionados aos conceitos de masculinidade e feminilidade. ${ }^{7} \mathrm{Em}$ se tratando destes constructos, Melo; Giavoni ${ }^{8}$ ressaltam que eles são elaborados a partir do momento que o indivíduo incorpora em seu autoconceito alguns traços, normas, papéis e valores condizentes aos constructos de masculinidade e feminilidade.

Porém, os indivíduos podem diferir em relação aos esquemas de gênero, apresentando o esquema masculino ou o feminino, bem como, podem apresentar os dois esquemas ou serem aesquemáticos. ${ }^{9}$ No ponto de vista de Giavoni; Tamayo, ${ }^{10}$ os indivíduos podem ser heteroesquemáticos masculinos (HM), os que apresentam predominância do esquema masculino, heteroesquemáticos femininos (HF), predominância para o esquema feminino e isoesquemáticos (ISO-E) os que apresentam uma simetria entre os dois esquemas.

Além destes constructos, outro que pode apresentar grande influência no desempenho dos atletas é o perfil idiocêntrico-alocêntrico, que está diretamente relacionado a teoria do individualismo/coletivismo.
No que tange esta teoria, Triandis ${ }^{11,12}$ destaca que as diferentes culturas na sociedade podem formar, tanto indivíduos individualistas, como coletivistas, designando de Idiocêntrico os indivíduos com traços individualistas e de Alocêntrico, os indivíduos com traços coletivistas. Os indivíduos idiocêntricos apresentam um autoconceito independente de seus grupos de pertença, preocupando-se mais com a realização do eu individual, enquanto, os indivíduos alocêntricos enfatizam mais a integridade do grupo e apresentam um autoconceito interdependente..$^{11,13}$

Em relação ao Perfil Idiocêntrico-Alocêntrico, Melo; Giavoni ${ }^{8}$ destacam que, quando separados por grupos, os atletas tendem a apresentar características de personalidade semelhantes, ou quando muito, diferem quanto à intensidade, porém, esta autora ressalta que não existe um perfil psicológico ideal para atletas.

No esporte de alto rendimento, estímulos relacionados aos indicadores psicossociais podem causar efeitos positivos ou negativos que influenciam diretamente o desempenho dos atletas, uma vez que o esporte é construído socialmente. Diante do exposto, o objetivo geral deste estudo é investigar qual o perfil dos atletas de jiu-jitsu de Chapecó-SC, em relação aos esquemas de gênero e o perfil idiocêntrico-alocêntrico.

\section{MÉTODO}

Este estudo fez parte de um projeto de pesquisa maior intitulado "Perfil esportivo e artístico de atletas e bailarinos" devidamente submetido e aprovado junto ao Comitê de Ética em Pesquisas com Seres Humanos da Universidade do Estado de Santa Catarina (UDESC) sob o número de protocolo 275.381/2013. Trata-se de um estudo não probabilístico, com corte transversal, descritivo de abordagem quali-quantitativa. ${ }^{14}$

Participaram do presente estudo, 32 praticantes de jiu-jitsu, do sexo masculino, jovens e adultos que frequentam uma academia de jiu-jitsu no município de Chapecó, localizado na região oeste de Santa Catarina. Para inclusão dos praticantes no estudo foram utilizados os seguintes critérios: ter idade maior ou igual a 16 anos e menor que 60 anos, ser praticante de jiu-jitsu no mínimo há um ano e participar de competições regionais, estaduais, nacionais e/ou internacionais. Para participar do estudo todos os praticantes de jiu-jitsu assinaram o termo de consentimento livre e esclarecido. Os praticantes menores de 18 anos apresentaram o termo assinado pelos pais ou responsáveis.

Como instrumentos foram aplicados três questionários: o primeiro foi relacionado às características sociodemograficas, econômicas e sociais, as quais foram categorizadas da seguinte forma: origem, cor da pele (preta, branca, amarela, parda e indígena); nível econômico (alto, médio e baixo); estado civil (com companheiro e sem companheiro); grau de escolaridade (analfabeto, ensino fundamental I e II, ensino médio e ensino superior); tempo de prática; frequência semanal das aulas; duração das aulas; e nível das competições (regional, estadual, nacional e internacional). As questões referentes às características dos lutadores e do treinamento foram elaboradas pela própria autora. As características sociodemográficas foram de acordo com 
o Instituto Brasileiro de Geografia e Estatística/IBGE ${ }^{15}$ e as características econômicas com a Associação Brasileira de Empresas de Pesquisas/ABEP. ${ }^{16}$

O segundo foi o Inventário Masculino dos Esquemas de Gênero do Autoconceito (IMEGA) proposto por Giavoni; Tamayo ${ }^{17}$ foi utilizado para avaliar os esquemas de gênero do autoconceito masculino. Este instrumento é composto por 71 itens, sendo 41 representantes do esquema masculino (escala masculina) e, 30 representantes do esquema feminino (escala feminina). Os fatores da escala masculina são Egocentrismo, Ousadia, Racionalidade e da escala feminina são: Integridade, Sensualidade, Insegurança, Emotividade, Sensibilidade (fator de segunda ordem). Os itens, do IMEGA foram avaliados através de uma escala de cinco pontos, em que o escore zero indica que o item não se aplica ao respondente até o escore quatro, indicando que o item se aplica totalmente ao respondente. Por fim, os indivíduos foram classificados em Heteroesquemáticos Masculinos, Heteroesquemáticos Femininos e Isoesquemáticos.

Para avaliação da orientação esportiva (individualismo e coletivismo) foi utilizado o questionário referente ao Inventário de Perfil Idiocêntrico-Alocêntrico de Atletas (Perfil I-A), validado por Melo, Giavoni ${ }^{18}$ sendo composto por 27 itens, os quais se subdividem em três fatores para o Idiocentrismo: Auto-Realização e Competitividade, Hedonismo, Distância Emocional de Equipe e um fator de segunda ordem - Nível de Idiocentrismo e um fator para o Alocentrismo: Nível de Alocentrismo. Estes itens também foram avaliados através de uma escala de cinco pontos, onde o escore zero indica que o item não se aplica ao respondente até o escore quatro, indicando que o item se aplica totalmente ao respondente. Por fim, os indivíduos foram classificados em Heteroidiocêntricos, Heteroalocêntricos e Isocêntricos.

Os dados foram tabulados e analisados no programa estatístico SPSS versão 20.0, utilizando os recursos de estatística descritiva: frequências (relativa e absoluta), média e desvio padrão.

\section{RESULTADOS}

Os resultados apontam que a média de idade dos praticantes de jiu-jitsu participantes deste estudo é de 27,5 anos $(d p=6,10)$, os quais treinam em média há $4,56$ anos ( $d p=4,39)$ com uma frequência semanal de 3,50 dias $(d p=0,91)$ e com uma duração de 1,59 horas $(d p=3,64)$.

A tabela 1 apresenta às características sociodemográfica e esportivas dos praticantes de jiu-jitsu investigados, em que 100\% residem em Chapecó (SC); 65,6\% $(n=21)$ vivem com uma companheira, 75\% ( $n=24)$ se declararam de cor branca e $75 \%(n=24)$ estão inseridos na classe média.

Em relação aos esquemas de gênero do autoconceito, observa-se na tabela 2 , que $62,5 \%(n=20)$ dos praticantes de jiu-jitsu apresentam uma predominância para o perfil isoesquemático, o qual demonstra uma simentria entre os esquemas masculino e feminino. No entanto, quando observado os demais perfis, percebe-se que $10 \%(n=10)$ apresentam maior predomínio no perfil heteroesquemático masculino e $2 \%(n=02)$ no heteroesquemático feminino.

No que diz respeito ao Perfil Idiocêntrico-Alocêntrico percebe-se nesta mesma tabela que $65,6 \% \quad(n=$ 21) dos praticantes classificaram-se como alocêntricos, o que significa que estes praticantes são mais coletivistas e $34,4 \%(n=11)$ como isocêntrico, não aparecendo nenhum praticante idiocêntrico, o que chamou a atenção pelo fato desta modalidade ser individual.

Tabela 1 - Características sociodemográficas e esportivas dos atletas de Chapecó da modalidade de jiu-jitsu.

\begin{tabular}{llcc}
\hline \multicolumn{2}{c}{ Variáveis } & N & $\%$ \\
\hline Origem & Chapecó & 32 & 100 \\
\hline Estado civil & Sem companheiro (a) & 11 & 34,4 \\
& Com companheiro (a) & 21 & 65,6 \\
\hline Cor de pele & Preta & 03 & 9,4 \\
& Branca & 24 & 75,0 \\
& Amarela & 05 & 15,6 \\
\hline Classe & Baixa & 01 & 3,1 \\
& Média & 24 & 75,0 \\
& Alta & 07 & 21,9 \\
\hline
\end{tabular}

$n=$ Frequência absoluta. \% = Frequência relativa

Tabela 2 - Esquemas de Gênero do Autoconceito e Perfil Idiocêntrico-Alocêntrico dos atletas Masculinos de Chapecó da modalidade de jiu-jitsu.

\begin{tabular}{lcc}
\hline Esquemas de Gênero & $\mathbf{N}$ & $\%$ \\
\hline ISOE & 20 & 62,5 \\
HF & 2 & 6,3 \\
HM & 10 & 31,3 \\
\hline Perfil I-A & $\mathbf{N}$ & $\%$ \\
\hline ISOC & 11 & 34,4 \\
ALO & 21 & 65,6 \\
\hline
\end{tabular}

n= frequência absoluta, \% = frequência relativa; HM: Heteroesquemático masculino; HF: Heteroesquemático feminino; ISOE: Isoesquemático; ISOC: Isocêntrico, ALO: Alocêntrico

\section{DISCUSSÃO E CONSIDERACִÕES FINAIS}

O objetivo do presente estudo foi investigar o perfil dos praticantes de jiu-jitsu em relação aos esquemas de gênero e o perfil idiocêntrico e alocêntrico para melhor compreender o comportamento dos atletas desta modalidade. Os atletas demonstraram em sua maioria pertencerem, às classes médias e altas, o que vem ao encontro do exposto por Andrade et al. ${ }^{19}$ que avaliou atletas de jiu-jitsu, na cidade de Florianópolis (SC) e verificou que a maioria pertenciam a classe alta e obtinham ensino superior. Esse resultado pode ser explicado pela realidade deste esporte, o qual normalmente é praticado em locais particulares.

Com relação aos esquemas de gênero do autoconceito, observa-se que os praticantes de jiu-jitsu em sua maioria, apresentam perfil isoesquemático. Gomes, Sotero; Giavoni ${ }^{20}$ esclarecem que os isoesquemáticos apresentam desenvolvimento proporcional dos esquemas masculino e feminino, equilibrando as tendências individualistas de poder, autosuperação e domínio. Além disto, os isoesquemáticos são portadores dos dois esquemas de gênero, masculino e feminino, os quais desenvolvem fatores como o egocentrismo, ousadia e racionalismo, bem como integridade, sensualidade, in- 
segurança, emotividade, sensibilidade. ${ }^{20}$ Fatores estes, que influenciam no comportamento de um lutador de jiu-jitsu, já que configura um estilo de luta com quedas, traumatismos, torções, projeções, imobilizações, chaves e estrangulamentos, além das solicitações de ataque e esquiva. ${ }^{4}$

Neste âmbito, os autores acreditam que esta simetria dos esquemas masculino e feminino, existente na maioria dos lutadores participantes deste estudo, é condizente com a especificidade da luta, e isto pode auxiliar os resultados individuais e por equipe, tanto nos treinamentos como nas competições.

Em relação ao perfil idio-alocêntrico, os lutadores apresentam-se, em geral, como alocêntricos, indivíduos com um predomínio do coletivismo, o que surpreende pelo fato do jiu-jitsu ser um esporte individual. Convém ressaltar que um atleta com perfil alocêntrico apresenta um self interdependente, grande preocupação pela coesão e bem-estar da equipe, além de traços como lealdade, fidelidade, submissão, responsabilidade e resignação. Além disto, de modo geral, os atletas alocêntricos, sentem-se ajustados socialmente, reduzindo assim, a necessidade de utilizarem artifícios físicos e psíquicos para impressionar e se destacar diante dos demais. ${ }^{8}$

Traços estes, muito presentes na rotina de um lutador de jiu-jitsu, pois além da disputa individual no tatame, há uma valorização pela integridade do grupo, em que muitas vezes, o lutador acaba sendo submisso a proposta da sua equipe diante do objetivo que a mesma busca na competição. Ou seja, além do lutador buscar o mais alto lugar no pódium individualmente, ele deve se preocupar com os demais membros da sua equipe, pois quanto mais atletas subirem ao pódium, melhor o resultado final da mesma. Esta busca por melhores resultados visando a equipe, se deve a uma regra do jiu-jitsu a qual ressalta que os resultados dos três primeiros colocados de cada categoria, valem pontos para a disputa por academias dentro da competição. ${ }^{21}$

Diante do exposto não foi encontrado nenhum estudo com estes constructos referindo-se aos lutadores de Jiu- Jitsu em específico, o que dificultou uma discussão mais abrangente sobre o tema em questão. Por isso, é importante a realização de mais estudos com direcionamento aos atletas de esportes de combate, para assim buscar novos rumos no que diz respeito aos aspectos psicológicos dos mesmos; deste modo ajudando-os a melhorar o seu desempenho e o planejamento de treinamento por parte da equipe técnica.

Este estudo descritivo contou com poucos atletas e não controlou o desempenho esportivo dos atletas participantes. Assim, sugere-se que outros estudos possam ser realizados para confirmarem as características psicológicas aqui descritas para os praticantes da modalidade de forma geral, bem como identificar possíveis influencias culturais nos traços psicológicos aqui estudados.

Através do presente estudo, é possível concluir que os lutadores de jiu-jitsu do município de Chapecó, apresentam um predomínio para o perfil isoesquemático, em que os mesmos desenvolvem fatores do esquema masculino e feminino relevantes nos momentos de treinamento e competições.

No que tange ao perfil Idiocêntrico-Alocêntrico há uma predominância para o Perfil Alocêntrico, deixando claro que a prática desse esporte, permite que o atleta participe e se preocupe com o grupo, ajudando assim, a meIhora do desempenho da equipe e não somente do individuo.

Espera-se que estes resultados possam auxiliar em novas propostas de treinamento no que diz respeito aos lutadores de jiu-jitsu do município de Chapecó-SC, buscando assim, melhores resultados nas competições.

\section{REFERÊNCIAS}

1. Carballido LGG. Uma aproximación prática a la psicologia del deporte. Rev. Digital - Buenos Aires - Año 7. 2001. 11. Disponível em:<http://www.efdeportes.com/>

2. Junior HS, Oliveira SRS, Simões AC. Avaliação das Tendências Competitivas de Atletas de Judô: uma analise entre gêneros. Maringá 2009;20(4):519-28. doi: 10.4025/ reveducfis.v20i4.6353.

3. Bara Filho MG, Ribeiro LCS, Garcia FG. Comparação de características da personalidade entre atletas brasileiros de alto-rendimento e indivíduos não-atletas. Rev Bras Medic Esporte 2005;11(2):115-20. doi: 10.1590/S151786922005000200004.

4. Ide BN, Padilha DA. Possíveis lesões decorrentes da aplicação das técnicas do jiuj itsu desportivo. Lecturas Educación Física y Deportes 2005;10(83):6.

5. Bravo E. Jiu Jitsu sem mistérios, tradução Larissa Wostog Ono. São Paulo: Modras, 2011.

6. Brandt R. Percepções de um medalhista olímpico acerca da importância dos estados de humor no rendimento esportivo. 2012.

7. Bem SL. Gender schema theory: a cognitive account of sex typing. Psychological Review 1981;88(4):354-64. doi: 10.1037/0033-295X.88.4.354.

8. Melo GFD, Giavoni A. O perfil psicológico de atletas baseado na teoria do individualismo e do coletivismo. Rev Bras Psicol Esporte 2010;3(1):2-18.

9. Markus H. Self-schemata and processing information about the self. Journal of personality and social psychology 1977;35(2):63. doi: 10.1037/0022-3514.35.2.63.

10. Giavoni A, Tamayo Á. The psychological synthesis evaluated by the Interactive Model. Psicologia: Reflexão e Crítica 2010;23(3):593601. doi: 10.1590/S0102-79722010000300020.

11. Triandis HC. The self and social behavior in differing cultural contexts. Psychological review 1989;96(3):506. doi: 10.1037/0033-295X.96.3.506.

12. Triandis HC. Individualism \& collectivism. Westview press. 1995.

13. Triandis HC, Leung K, Villareal MJ,Clack FI. Allocentric versus idiocentric tendencies: Convergent and discriminant validation. Journal of Research in personality 1985;19(4):395415. doi: 10.1016/0092-6566(85)90008-X.

14. Gaya A. Ciências do movimento humano: introdução à metodologia da pesquisa. Artmed Editora. 2009.

15. Brasil. IBGE. Instituto Brasileiro de geografia e Estatística. Censo Demográfico 2000. Características Étnico-raciais da População: Um estudo das categorias de classificação de cor ou raça. 2008. Recuperado em 10 de março, 2015. Disponível em: http://www.ibge.gov.br/home/estatistica/ populacao/caracteristicas_raciais/PCERP2008.pdf

16. Associação Brasileira de Empresas de Pesquisa/ABEP. Critério de Classificação Econômica Brasil. 2013. Acessado em: 10/3/2015. http://www.abep.org/new/codigosCondutas.aspx

17. Giavoni A, Tamayo A. Masculine inventory of the self- 
concept's gender squemas. Psicologia: Teoria e Pesquisa 2003;19(3):249-59.

18. Melo GFD, Giavoni A. Elaboration and Validation of the athletes idiocentric and allocentric profile inventory (IA Profile). Span J Psychol 2010;13(02):1021-31. doi: 10.1017/ S1138741600002663.

19. Andrade A, Crocetta TB, Silva RB, Casagrande PO, Pruner L, Gallo SKAM, Viana MS, Abreu LC. Tempo de reação, motivação e caracterização sociodemográfica de atletas iniciantes de Jiu-Jitsu. Ciência e Movimento 2014;22(1):111
21. doi: 10.18511/0103-1716.

20. Gomes SA, Sotero RDC, Giavoni A, Melo GFD. Body composition and physical fitness level evaluation among futsal athletes classified into gender schemas typological groups. Revista Brasileira de Medicina do Esporte 2011;17(3):156-60. doi: 10.1590/S1517-86922011000300001.

21. Brasil. International brazilian Jiu-Jitsu Federation (IBJJF). Livro de regras e regulamento geral de competições. Manual de formatação de competições. Rio de Janeiro, 2015;04.

Como citar: VIEIRA, Mariluce Poerschke et al. Esquemas de gênero e perfil idiocentrico e alocentrico dos lutadores de Jiujitsu de Chapecó-SC. Cinergis, Santa Cruz do Sul, v. 17, n. 3, set. 2016. ISSN 2177-4005. Disponível em: <https://online. unisc.br/seer/index.php/cinergis/article/view/7832 >. Acesso em: 30 sep. 2016. doi:http://dx.doi.org/10.17058/cinergis. v17i3.7832. 